\title{
Multilamellar spherical particles as potential sources of excessive light scattering in human age-related nuclear cataracts
}

\author{
M. Joseph Costello ${ }^{1}$, Sönke Johnsen ${ }^{2}$, Sangeetha Metlapally ${ }^{1}$, Kurt O. Gilliland ${ }^{1}$, Lesley \\ Frame $^{3}$, and Dorairajan Balasubramanian 4 \\ ${ }^{1}$ Department of Cell and Developmental Biology, University of North Carolina, Chapel Hill, NC \\ 2 Department of Biology, Duke University, Durham, NC \\ ${ }^{3}$ Department of Materials Science and Engineering, University of Arizona, Tucson, AZ \\ ${ }^{4}$ L.V. Prasad Eye Institute, Hyderabad, India
}

\begin{abstract}
The goal of this project was to determine the relative refractive index (RI) of the interior of multilamellar bodies (MLBs) compared to the adjacent cytoplasm within human nuclear fiber cells. MLBs have been characterized previously as 1-4 $\mu \mathrm{m}$ diameter spherical particles covered by multiple lipid bilayers surrounding a cytoplasmic core of variable density. Age-related nuclear cataracts have more MLBs than transparent donor lenses and were predicted to have high forward scattering according to Mie scattering theory, assuming different RIs for the MLB and cytoplasm. In this study quantitative values of relative RI were determined from specific MLBs in electron micrographs of thin sections and used to calculate new Mie scattering plots. Fresh lenses were Vibratome sectioned, immersion fixed and en bloc stained with osmium tetroxide and uranyl acetate, or uranyl acetate alone, prior to dehydration and embedding in epoxy or acrylic resins. Thin sections $70 \mathrm{~nm}$ thick were cut on a diamond knife and imaged without grid stains at $60 \mathrm{kV}$ using a CCD camera on a transmission electron microscope (TEM). Integrated intensities in digital electron micrographs were related directly to protein density, which is linearly related to RI for a given substance. The RI of the MLB interior was calculated assuming an RI value of 1.42 for the cytoplasm from the literature. Calculated RI values for MLBs ranged from 1.35 to 1.53. Thus, some MLBs appeared to have interior protein densities similar to or less than the adjacent cytoplasm whereas others had significantly higher densities. The higher density MLBs occurred preferentially in older and more advanced cataracts suggesting a maturation process. The bilayer coats were more often observed in MLBs from transparent donors and early stage cataracts indicating that bilayer loss was part of the MLB maturation, producing large low-density spaces around dense MLB cores. These spaces were frequently observed in advanced cataracts from India as large low-density crescents and annular rings. Predicted scattering from Mie plots using particles with dense cores and low-density rims was higher than reported previously, although the most important factor was the relative RI , not the MLB coat or lack thereof. In conclusion, the
\end{abstract}

(C) 2010 Elsevier Ltd. All rights reserved.

Corresponding author: Dr. M. Joseph Costello, Ph.D., UNC-Chapel Hill School of Medicine, Cell and Developmental Biology, 232 Taylor Hall, CB\# 7090, Chapel Hill, NC, 27599, Phone: (919) 966-6981; FAX: (919) 966-1856; mjc@ med.unc.edu .

Publisher's Disclaimer: This is a PDF file of an unedited manuscript that has been accepted for publication. As a service to our customers we are providing this early version of the manuscript. The manuscript will undergo copyediting, typesetting, and review of the resulting proof before it is published in its final citable form. Please note that during the production process errors may be discovered which could affect the content, and all legal disclaimers that apply to the journal pertain. 
measurements confirm the high protein density and RI of some MLB interiors compared to adjacent cytoplasm. This high RI ratio used in the Mie calculations suggests that for 2000 MLBs/ $\mathrm{mm}^{3}$, about half that reported for early stage nuclear cataracts from the US, the forward scattering could be more than $30 \%$ of the incident light. Therefore, the extent of forward scattering and its influence on macular visual acuity could be important components of ophthalmological evaluations of cataract patients.

\section{Keywords}

Mie scattering theory; nuclear cataracts; refractive index; electron microscopy; lens ultrastructure

\section{Introduction}

Multiple sources of light scattering have been identified in human age-related nuclear cataracts. Modifications to cytoplasmic crystallin proteins result in protein aggregation and produce density and refractive index fluctuations mainly in the range of 20-200 nm that contribute to retinal stray light and backscatter detectable by slit lamp examination (Benedek, 1997; Costello and Kuszak, 2008; Datiles et al., 2002; Metlapally et al., 2008; Truscott, 2005). Damage to nuclear fiber cell membranes produces defects in a similar size range, especially low-density regions adjacent to membranes, that are predicted to contribute significant scattering at low and high angles as nuclear cataracts become more advanced (Al-Ghoul and Costello, 1996; Costello et al., 2008). Globular particles predicted to be present from theoretical analyses of human lens light scattering (van den Berg, 1997) were discovered in ultrastructural studies and characterized as MLBs containing a core of cytoplasmic proteins surrounded by multiple lipid-rich bilayers forming a coat (Gilliland et al., 2001). These 1-4 $\mu \mathrm{m}$ diameter coated particles were observed in every early age-related nuclear cataract examined from the US with an average density of $4000 \mathrm{per} \mathrm{mm}^{3}$ and a maximum density of 20000 per $\mathrm{mm}^{3}$ (Gilliland et al., 2004). Similar MLB densities were observed in advanced nuclear cataracts from India and one Indian cataract displayed about 400000 per $\mathrm{mm}^{3}$ (Gilliland et al., 2008).

Mie light scattering theory was employed previously to calculate the potential scattering from MLBs. For nuclear cataracts from the US, the average US cataract was predicted to scatter about $20 \%$ of the incident light (Costello et al., 2007) and for an advanced nuclear cataract from India having a large number of MLBs, $100 \%$ scattering, or total opacification, was predicted (Gilliland et al., 2008). These Mie calculations were based on the assumption that the interior RI of the MLBs was 1.49 compared to a value of 1.40 for the cytoplasm from the literature (Pierscionek, 1997). The ratio 1.49/1.40 was a key input parameter for the Mie calculations. An objective of this study was to make quantitative determinations of the RI ratio directly from TEM images of thin sections.

Over forty years ago, Bahr and Zeitler (1965) demonstrated that the electron microscope could produce images in which the intensity of scattered electrons recorded on film was directly related to mass density within the specimen. Subsequently, Lamvik and Davilla (1989) showed that path length through standard particles, such as polystyrene spheres of known constant density, could be used to confirm directly the accuracy of the mass density measurements in images of particles recorded on film. By integrating intensities in the digitized film along different path lengths through the spheres, the mass could be determined, as long as the film density was within the linear intensity response region. Modern CCD cameras, which simplify the recording of digital images, have been used here to determine integrated intensities of MLBs in relation to adjacent cytoplasm in TEM images of thin sections of constant thickness. Because protein mass density is directly 
related to RI, the relative RI of the MLBs and adjacent cytoplasm could be estimated from electron micrographs. For these measurements, we chose to use a recent determination of the average RI of 1.42 for aged human lens nuclei to represent the RI of the cytoplasm (Jones et al., 2005). The measured RIs from some of the MLB interiors were in excess of 1.51 , thus supporting the previously assumed values (Costello et al., 2007). New images of MLB ultrastructure indicate a maturation process as the cores of the MLBs increase in density during aging and cataract formation. Additional Mie calculations of the predicted scattering suggested that a large portion of the incident light could be scattered in the forward direction and have an adverse effect on image formation at the macula even for nuclear cataracts in early stages of formation.

\section{Materials and Methods}

\subsection{Lenses}

Age-related cataractous lens nuclei were obtained after extracapsular surgery as described in Gilliland et al., 2004 and Metlapally et al., 2008. From the US, eight nuclei 56-85 years old were obtained with grades 1-3 (on a 0-4 nuclear cataract scale), and from India, 10 nuclei 38-75 years old were obtained with grades 3-4, considered to be advanced nuclear cataracts from patients who were blind at the time of cataract surgery. Transparent donor lenses examined had an age range 34-71 years for 8 lenses from the US and 48-78 years for 5 lenses from India. All lenses were obtained according to the tenets of the Declaration of Helsinki, following procedures reviewed by the Institutional Review Board for protection of human subjects.

\subsection{Sample preparation and transmission electron microscopy}

Vibratome sections approximately $200 \mu \mathrm{m}$ thick were cut from fresh lenses (Fig. 1A) and processed as described previously (Gilliland et al., 2004). Briefly, Vibratome sections were immersion fixed for $12-18 \mathrm{~h}$ in $2.5 \%$ glutaraldehyde, $2 \%$ paraformaldehyde and $1 \%$ tannic acid in $0.1 \mathrm{M}$ cacodylate buffer ( $\mathrm{pH}$ 7.2). Fixed sections were washed with $0.1 \mathrm{M}$ cacodylate for three 15-min washings, treated with cold $0.5 \%$ osmium tetroxide for $60 \mathrm{~min}$, washed with deionized distilled water for three 15-min washings, washed once with $50 \%$ ethanol for $5 \mathrm{~min}$, stained in $2 \%$ uranyl acetate (in 50\% ethanol) in the dark for $30 \mathrm{~min}$ and dehydrated through a graded ethanol series. Sections were infiltrated and embedded in an epoxy resin (Epon 812, EMS, Hatfield, PA). For some sections, the osmium tetroxide step was omitted and sections were embedded in acrylic resin (LR White, EMS, Hatfield, PA). Although the alternative post fixation and embedding is not ideal for membranes, it is commonly used to improve accessibility of epitopes for antibody labeling. The main goals for this study were to avoid specific labeling of proteins by osmium tetroxide and to take advantage of the excellent preservation of cytoplasmic protein in LR White resin. Thin sections (70 nm) were cut with a diamond knife (Diatome US, Hatfield, PA) from mesas raised to include the embryonic and fetal nuclei. Thin sections were typically mounted on 300 mesh hexagonal copper grids (Fig. 1B; T300H-Cu, EMS, Hatfield, PA) that have thin grid bars and give high transmission. It is important to note that the thin sections mounted on the grids were not stained because of the potential for uneven deposition of a relatively large amount of heavy metals on the cut surfaces of the sections.

The low contrast en bloc stained thin sections were viewed at $60 \mathrm{kV}$ on a FEI-Philips Tecnai 12 TEM (FEI Company, Hillsboro, OR) equipped with a Gatan 794 bottom mounted slow scan $1 \mathrm{k} \times 1 \mathrm{k}$ CCD digital camera (Pleasanton, CA). The CCD detector was fiber-optically linked to a high resolution YAG crystal scintillator. The CCD camera was controlled by Digital Micrograph (version 3) and Digital Montage software (Gatan, Pleasanton, CA) that used controlled beam shifting to record large fields of view even at high magnification. The 
objective aperture was $40 \mu \mathrm{m}$ in diameter. Low-dose imaging software was used to measure the electron dose and minimize the exposure to the beam-sensitive thin sections. Initial images were recorded with an electron dose less than $400 \mathrm{e}^{-} / \mathrm{nm}^{2}\left(2 \mathrm{~s}\right.$ exposure at $200 \mathrm{e}^{-} /$ $\left.\mathrm{nm}^{2} \mathrm{~s}\right)$. After minimal dose images were recorded for quantitative measurements, additional images were recorded at normal intensity levels. Liquid nitrogen-cooled cold finger blades at the sample were always used to minimize contamination. Images were recorded ideally at a low magnification that permitted the MLB, adjacent cytoplasm, a hole (no sample) and a grid bar to appear in the montage. The hole, which is commonly a tear in the embedding resin, is essential to establish the intensity reading where no electrons are absorbed or scattered (near 255 on a $0-255$ image density gray scale). In some cases it was not possible to include a large hole in the field of view so images were recorded in less than ideal conditions, for example, with a small hole adjacent to the MLB or in the clear shell of the MLB where some embedding resin remains. These images were analyzed separately from those recorded under the more ideal conditions.

\subsection{Image processing and statistical analysis}

The formation of an image from transmitted and scattered electrons is a statistical process. Electrons strike the crystal scintillator, creating photons that follow a fiber optic light path to the CCD detector where they are recorded with an average pixel size of $24 \mu \mathrm{m}$. At low electron doses, only a fraction of the electrons are captured and a fraction of the pixels record intensity. Even for lightest regions of the image (lowest electron intensity) where there is no sample, not all of the pixels will be at the maximum and some statistical fluctuation is evident. The CCD camera, although Peltier cooled, also has a small amount of noise (less than $0.5 \%$ over the whole image) that is evident by examining individual pixels in images of grid bars, which should pass no electrons (highest electron density). Therefore, the pixels must be averaged over a sufficiently large area to smooth out the statistical fluctuations and produce integrated intensities.

Images are initially converted to an 8-bit format (0-255 scale) using ImageJ (version 1.39u, Rashband, NIH) and each image then represents precise values that can be used to derive integrated intensity data. The small variability in measuring integrated intensities occurs in choosing the size and location of the selected region using the ellipse selection tool in ImageJ. Two procedures were used. The whole MLB interior was selected (avoiding membranes and density gradients of the coat) and identical selections taken in the adjacent cytoplasm within the same cell and in a hole. Alternatively, a small ellipse was used to sample the MLB interior in five locations and these values were averaged and compared to similar measurements in adjacent cytoplasm and a hole. Two measurements for each of the 61 images of MLBs were made by independent investigators and the results were averaged. The integrated intensities and calculated RIs were made to four significant figures and rounded to three. Consistent with the standard deviations of 1-2\%, the resulting calculated RIs for MLBs were reported with a precision of about \pm 0.01 . Once the images were recorded, any manipulation of the pixels with software image analysis programs, such as gamma, brightness, contrast or sharpness adjustments, were avoided because these changes are artificial and may lead to erroneous RI calculations.

\subsection{Theoretical analysis}

The refractive indices of the cores of the MLBs were estimated from the electron micrographs in a two-step process. First, the staining intensity of the MLB core relative to that of the surrounding cytoplasm was determined by measuring the average gray scale values of similar sized samples of both regions, referred to hereafter as $G_{M L B}$ and $G_{c y t}$. These values were determined as integrated intensities from selected areas in ImageJ (version 1.39u, Rashband, NIH Image) summing the intensities of all of the selected pixels 
in the 8-bit image divided by the area of the selection region. Typically, the MLB selected area, the selected area of the adjacent cytoplasm and selected area in a hole (no sample) were the same size (ranging from about 200 pixels to over 100000 pixels total in the an elliptical region of interest depending on the magnification and the size of the MLB). The Gatan 794 camera that produced the electron micrographs is highly linear (less than 1\% nonlinearity over the whole dynamic range), so the gray scale at any given point is linearly proportional to the transmittance through the section. The offset of the camera was corrected for by subtracting the measured gray scale values from the gray scale value of a hole in the section $G_{\text {hole }}$ (which by definition has a transmittance of 100\%). Thus, the staining density of the MLB, $S_{M L B}$, normalized by that of the cytoplasm, $S_{c y t o}$, is:

$$
\frac{S_{M L B}}{S_{\text {cyto }}}=\frac{G_{\text {hole }}-G_{M L B}}{G_{\text {hole }}-G_{\text {cyto }}}
$$

This relative staining intensity can then be used to calculate the RI of the MLB core, $n_{M L B}$, if two assumptions are met. First, staining density must be linearly proportional to the actual density of the en bloc stained section. In order for this to be true, the section has to have uniform thickness and even stain-uptake, which appears to occur in thin sections cut with a diamond knife where compression and knife marks are avoided. The stain also has to be non-selective. While electron microscopy stains are fairly non-selective, they are not perfectly so. However, in this case, the stain is always acting on the same material (cytoplasmic protein either inside or outside an MLB), so any selectivity of staining is unlikely to be significant. Finally, the staining cannot be too intense. In general, the relationship between the density of an absorbing substance and light transmission is exponential, but all exponential curves are linear for small values of intensity. In these electron micrographs, the staining is so light that it is likely well within the linear region. For all these reasons, we are confident that staining density is linearly proportional to the actual density of the underlying en bloc stained cytoplasm, both inside and outside the MLBs.

The second assumption is that the density of the en bloc stained cytoplasm is linearly proportional to its refractive index minus one, and that the coefficient of this relationship is the same for both MLB and surrounding cytoplasm. While density and refractive index are not correlated in general, they are usually linearly correlated for a given material over a reasonable range of densities. The coefficient of the relationship between density and $n-1$ is known as the refractive index increment and is considered a fundamental property of a material. Linear relationships have been reported for sucrose and matrizamide used to form density gradients (Birnie et al., 1974). A linear relationship has been reported for lens proteins, $\mathrm{n}-1=0.19 \rho$ (Pierscionek et al., 1987). Again, because the material both inside and outside the MLB is cytoplasm, the refractive index increment is likely to be the same.

Since the absolute densities of the cytoplasm and MLB are unknown, it is not possible to directly calculate the refractive index increment. However, because all the relationships are linear, all we need to know is the index of the surrounding cytoplasm, $n_{\text {cyto }}$, and the relative staining density. Thus:

$$
n_{M L B}=1+\frac{S_{M L B}}{S_{c y t o}}\left(n_{c y t o}-1\right)
$$

This equation is linear and gives the correct answer for $S_{M L B}=0\left(n_{M L B}=1\right)$ and $S_{M L B}=S_{c y t o}$ $\left(n_{M L B}=n_{c y t o}\right)$. Since two points uniquely define a line, this equation is most likely correct. 
The index of the surrounding cytoplasm was assumed to be 1.42 based on results from Jones et al., 2005. While this value may not be exact, it has little effect on the scattering calculations, because these depend on the relative refractive index $\mathrm{m}=n_{M L B} / n_{\text {cyto }}$. For example, if $S_{M L B} / S_{\text {cyto }}=1.1$, then $m=1.0296$ for $n_{\text {cyto }}=1.42$. If $n_{\text {cyto }}$ instead actually equaled 1.40 or $1.44, m$ would only change to 1.0286 and 1.0306 , respectively, neither of which have any significant effect on light scattering.

\section{Results}

\subsection{Maturation of MLBs}

The ultrastructure of MLBs observed in transparent and early stage nuclear cataracts often reveal multiple thin bilayers in a coat or shell (Fig. 2A,B), as noted previously (see Fig. 9 in Gilliland et al., 2001, Fig. 4 in Gilliland et al., 2004, Fig. 2A in Costello et al., 2007 and Figs. 4A,D in Gilliland et al., 2008). The 3-10 layers are each about $5 \mathrm{~nm}$ thick, typical of pure lipid bilayers but not sufficiently thick to contain the main fiber cell membrane proteins (Gilliland et al., 2001). Many MLBs display fragments of the lipid bilayer coats (Fig. 2C), consistent with previously reported images (see Figs. 8, 9 in Gilliland et al., 2001, Fig. 4 Gilliland et al., 2004 and Fig 2A in Costello et al., 2007). These fragments of membranes may represent a partial disruption during sectioning and sample preparation or the pattern may signal a stage of degeneration of the bilayer coat. Many MLBs in more advanced cataracts display no evident bilayer membranes in the shell surrounding the MLB core (Fig. 2D) even in epoxy embedded Vibratome sections stained with osmium tetroxide and uranyl acetate where the preservation of membranes is usually excellent. The bilayers or their fragments in such MLBs are not visible even at multiple tilt angles $\left( \pm 50^{\circ}\right)$ at high magnification $(30000 \times)$ suggesting that the bilayers have been lost during a maturation process. A gallery of MLBs shows a range of MLB densities and shell sizes (Fig. 3). In some MLBs the association with the fiber cell membrane is evident (Fig. 3A and 3E). In these MLBs it is reasonable to speculate that the lipid-rich bilayers of the coat were derived from the fiber cell membranes. In other MLBs that appear to have no obvious connection to the membranes, the shell is often large and the core is dense (Fig. 3D and 3F). This pattern can be interpreted as a maturation of the MLBs that includes a condensation of the core that accompanies the loss of the bilayer coat.

\subsection{Mature MLBs with high-density cores}

Two examples of MLBs are presented that illustrate the measurement techniques and the appearance of MLBs that have high calculated internal RI (Figs. 4 and 5). In both examples, a large well-defined spherical MLB is embedded within the uniformly stained cytoplasm of a fiber cell. The MLBs and several well-preserved fiber cells are separated from large holes by grid bars. At high magnification the MLBs have wide low-density coats and dense and uniformly stained cores (Figs. 4 inset and 5 inset) consistent with closely packed concentrated protein. The calculated RIs derived from measurements on the low magnification views are 1.49 and 1.53, respectively (Fig. 4 and 5).

\subsection{Distribution of MLB calculated RI values}

The calculated RI values of all of the MLBs measured are summarized in a histogram (Fig. 6). The MLBs that were considered to be ideal for the integrated intensity measurements, in which the images contained a large hole, as in Figs. 4 and 5, are shown in dark gray. Those MLBs in images that were less than ideal are shown in light gray. These images were still of high quality but contained only a small hole or no grid bar, as in Fig. 2C. There were a few MLBs in which the calculated RI and density were less than for the cytoplasm (see Fig. 3C). These were not commonly seen and it is not clear why they have low protein densities. It is noted that MLBs with low-density cores will still generate light scattering because the ratio, 
' $\mathrm{m}$ ', is the important parameter, not the absolute value of the core RI. If ' $\mathrm{m}$ ' is close to one, the Mie calculated results are similar for values of ' $m$ ' slightly larger or smaller than one.

\subsection{Calculated light scattering using the Mie scattering theory}

The Mie light scattering theory is appropriate for globular particles of any size and particularly well suited for MLBs in the average size range of 1-4 $\mu \mathrm{m}$ with variable internal refractive indices compared to the adjacent cytoplasm. Light scattering of coated particles was calculated for a wavelength of $550 \mathrm{~nm}$ and cytoplasmic RI of 1.42 (Jones et al., 2005) using the Mie theory for three conditions. First, the coat was assumed to contain 10 lipid bilayers, with a total thickness of $50 \mathrm{~nm}$ and with an RI of 1.50 (Matsuzaki et al., 2000), representative of the MLB in Fig. 2A, B. Second, the coat was assumed to be devoid of bilayers with an assumed RI of 1.35 typical of cytoplasm (as a dilute protein solution with water RI $=1.33$, Charney and Brackett, 1961) and a thickness of $200 \mathrm{~nm}$, similar to the MLB in Fig. 4 where the shell has a nearly uniform width. Third, the coat devoid of bilayers was assumed to be $350 \mathrm{~nm}$ with an RI of 1.35, representative of some MLBs, as in Fig. 5, where the shell was irregular with an average width of over $300 \mathrm{~nm}$. The resulting surface plots display the percent total scatter of the incident light for a wide range of MLB sizes and internal RIs, using a particle density of 2000 per $\mathrm{mm}^{3}$. This density is selected to be half of the average density from US cataracts and one tenth of the maximum density (Gilliland et al., 2004), accounting for the probability that only a fraction of the MLBs in a nuclear cataract will have the size and high internal RI to be strong sources of light scatter.

The scattering plots are exact calculations and are quite general. They do not depend on the properties of any particular MLB or the average properties of the observed MLBs. Thus, using the properties of the MLB in Fig. 2A as an example, the predicted scattering in Fig. $7 \mathrm{~A}$ (at radius $=1.6 \mu \mathrm{m}, \mathrm{RI}=1.43$ ) would be near the trough where the scattering is minimal (less than 5\% of incident light if all 2000 particles had these properties). If the MLB in Fig. $2 \mathrm{C}$ is representative (radius $=2.3 \mu \mathrm{m}, \mathrm{RI}=1.45$ ), the predicted scattering would be on the steep slope of the central peak in Fig. 7B (20\% scatter). If the MLBs in Figs. 4 (radius $=1.4$ $\mu \mathrm{m}, \mathrm{RI}=1.49$ ) or Fig. 5 (radius $=2.3 \mu \mathrm{m}, \mathrm{RI}=1.53$ ) are representative, then the predicted scattering would fall near the central peaks in Fig. 7B (30\% scatter) or 7C (35\% scatter), respectively. A real nuclear cataractous lens would have a distribution of particles with different properties. In principle the predicted scatter for each particle could be found from surface plots like those in Fig. 7 and the total scattering would be the sum of the scattering for all the particles. Alternatively, if an advancing cataract contained a large fraction of particles with the high internal RI $=1.49-1.53$, as in Fig. 6, then the predicted scattering would be near the maxima in Fig. 7 (greater than 30\%).

\subsection{Angular dependence of scattering using Mie scattering theory}

The Mie theory calculations also provide the complete description of the angular distribution of the scattered light. Because the main emphasis here is on large particles with high RI compared to the cytoplasm, the majority of light is scattered at low angles producing mainly forward scattering as described previously in Mie theory scattering plots as a function of average scattering angle (Costello et al., 2007). For instance, for an MLB with an internal RI only 0.02 units away from that of the cytoplasm, the scattering angle rapidly decreases to be confined to within about $15^{\circ}$ around the axis of the incident light (Costello et al., 2007). This is further emphasized in the series of normalized scattering curves for particles of different sizes (Fig. 8). As the particle radius increases in the range 0.5 to $4 \mu \mathrm{m}$, the average scattering angle rapidly decreases. The actual intensities (not normalized) on a relative scale illustrate that the total scattering of individual particles also increases dramatically as the size increases (Fig. 8). Thus, a $4 \mu \mathrm{m}$ diameter MLB that has an RI of 1.53 compared to 1.42 of the cytoplasm produces about 150 times more scattering than a similar MLB that is $1 \mu \mathrm{m}$ in 
diameter; the scattering angle at half peak height goes from about $7^{\circ}$ for the $4 \mu \mathrm{m}$ particle to about $20^{\circ}$ for the $1 \mu \mathrm{m}$ particle (Fig. 8). Forward scattering dominates the scattering distribution for MLBs described here.

\section{Discussion}

Human age-related nuclear cataracts develop slowly over decades usually beginning in the fourth to fifth decade of life after the onset of presbyopia (McGinty and Truscott, 2006). The underlying causes of lens changes during aging and nuclear cataract formation are thought to be largely a result of oxidative stress (Truscott, 2005). However, the effect of oxidation and other age-related factors on the internal structure of the lens fiber cells to produce objects that scatter light excessively is not well understood. We have employed ultrastructural techniques to identify the extent of cellular damage and theoretical analyses to evaluate the expected light scattering from each type of cellular defect.

Nuclear fiber cell membranes, cytoplasm and distinct scattering particles have been analyzed separately in previous studies conducted in this laboratory. Damage to fiber cells in lens nuclei includes the disintegration of fiber cells in situ, the accumulation of cellular debris (Al-Ghoul and Costello, 1996) and the breakdown and loss of components of the fiber cell membranes (Costello et al., 2008). In a series of studies using Fourier image analysis techniques, alterations in the dense packing of cytoplasmic crystallins were examined and it was found that the cytoplasm in nuclear fiber cells from cataracts was nearly indistinguishable from that in aged transparent donor lens nuclei even when the cataracts were advanced (Freel et al., 2002; Metlapally et al., 2008; Taylor and Costello, 1999). Distinctive spherical particles, the 1-4 $\mu \mathrm{m}$ diameter MLBs, were reported to be approximately 7.5 times more numerous in nuclear cataracts than in transparent donor lenses from the US (Gilliland et al., 2004). Based on the structural features of the MLBs and the assumed high RI of the core compared to the adjacent cytoplasm, Mie scattering theory was used to predict that the MLBs contributed significantly to forward scattering (Costello et al., 2007). In this study, quantitative electron microscopy was used to measure directly, from TEM images of thin sections, the relative RI of MLB cores compared to adjacent cytoplasm. If the cytoplasm was assumed to have an RI of 1.42 (Jones et al., 2005), then the MLB cores ranged from 1.35 to 1.53 with many MLB cores having values in the 1.47 to 1.53 range, as high as assumed in previous studies.

In this study the MLBs having dense cores with high RI were emphasized because these were the most commonly observed. Numerous images demonstrate that MLBs in transparent donor lenses and some early stage cataracts have MLBs with relative RI values near that of the cytoplasm (Figs. 2A, 3A-D) whereas many other MLBs in aged cataractous nuclei have dense core MLBs with high relative RI (Figs. 2D, 3F, 4, 5). This suggests a maturation process that is hypothesized to have several key steps. First, with age and cataract formation, the MLB core protein condenses to increase its density and decrease its volume. Next, the core appears to pull away from the cytoplasm causing the lipid bilayers of the coat to fragment (Fig. 2C). Finally, the lipid bilayers are degraded and lost leaving a wide clear coat of low RI (Figs. 2D, 3F, 4, 5). These changes are consistent with predicted gradual increased scattering as the particle interiors become denser and correlate with the observed slow development of age-related nuclear cataracts.

It is not known why a few MLB interiors produced relative RI values significantly lower than the assumed value of the cytoplasm of 1.42. One possible explanation is that the MLB coat enclosed a region of cytoplasm that contained a lower content of $\gamma$-crystallin, known to promote high-density packing of protein at low water content (Tardieu et al., 1992), resulting in a small enclosed region that retained water as the surrounding cytoplasm 
condensed during early development to produce the high RI nuclear region. Another explanation is that the aging of the MLB interior, isolated by the coat, can result in a variety of fates including disintegration leaving irregular fragments in the interior as reported previously (Gilliland et al., 2001). It should be pointed out that MLBs with low core RI scatter light nearly as strongly as those with high RI, because the critical parameter is the RI ratio of the core to the adjacent cytoplasm. When this ratio or its reciprocal is close to one, the scattering is similar.

Mie theory scattering plots illustrate the importance of specific structural features of the coated particles (Fig. 7). MLBs with thin high RI coats (50 nm, RI 1.50 for pure lipid bilayers) will scatter weakly for core RI values near 1.42 and scatter maximally for core RI values near 1.48 (Fig. 7A). MLBs with wide low RI coats (200-350 nm, RI 1.35 similar to cortical cytoplasm) will scatter weakly for core RIs $1.43-1.44$ and scatter maximally at $1.49-1.50$ (Figs. 7B,C). In spite of the small variations in the surface plots for different coat properties, the similarity of the Mie surface plots suggests that the important factors are the size of the MLB and the core RI, not the size or RI of the coat.

The absence of lipid bilayers within the coat of some dense core MLBs is difficult to prove conclusively. Extensive searching for bilayer profiles at high magnifications and various tilts failed to reveal any hints of lipids (see Figs. 2D) even when preservation of membranes and bilayers can be demonstrated in neighboring regions within the same sections or equivalent epoxy embedded sections stained with both osmium tetroxide and uranyl acetate to enhance visibility of lipids (Fig. 2A; also see Gilliland et al., 2001). The loss of membranes has been suggested previously to be potentially an initiating event in nuclear cataract formation, compromising the integrity of fiber cells and opening pathways for the redistribution of cytoplasmic proteins to form deposits in the extracellular space (Costello et al., 2008). It was suggested that the pure lipid components of plasma membranes and junctions might be subject to oxidative damage and this might also apply to the bilayers of the MLB coat (Costello et al., 2008).

The RI values of MLB cores relative to adjacent cytoplasm are derived directly from integrated intensities in digital electron micrographs. The magnification ideally was selected to be low enough to include the MLB within a fiber cell, a hole and a grid bar; magnification must also be high enough to have a sufficient number of pixels to give accurate integrated intensities with minimal statistical noise. Integrated intensities of whole MLB cores were compared to equivalent areas of adjacent cytoplasm of the same cell containing the MLB. This measurement process reduced the influence of most factors that could affect the intensity measurements. For example, the exact thickness of the thin section was not critical, only that the section did not change thickness in the region around the MLB, as would occur with knife marks or microtome compression bands. In addition, the accelerating voltage and objective aperture size, which both affect image contrast, were not critical; conditions were chosen to provide sufficient contrast to enable efficient searching for suitable MLBs. Furthermore, mass loss can be significant for thin sections especially at room temperature (Muller et al., 1992) and contamination can alter the mass in the imaged region. Although low dose exposure can minimize mass loss and cryo-blades around the specimen can reduce contamination, these factors are not critical, because cytoplasm and MLBs are affected similarly. Moreover, equivalent results were obtained with both epoxy and acrylic embedding media and using en bloc stains with or without osmium tetroxide, suggesting that observed differences in optical density reflect differences in protein density.

The measurement of the mass of biological components using the electron microscope has been conducted extensively based on early work of Zeitler and Bahr (1962; Bahr and Zeitler, 1965). The most common method is to deposit the biological material on a carbon film and 
employ the scanning transmission mode and darkfield imaging, using specialized instruments that give the mass per unit length of filaments and the mass per unit area of particles (Krzyzanek et al., 2009; Muller and Engel, 2001). Some important examples include the mass per unit length of myosin thick filaments (Lamvik, 1978), virus particles (Bahr et al., 1976), fibrinogen (Mosesson et al., 1981), two-dimensional crystals of aquaporin (Walz et al., 1994), amyloid fibrils (Kammerer et al., 2004) and vimentin intermediate filaments (Herrmann et al., 1996). These specimens have the advantage that they can be deposited on a substrate without stain, fixatives or embedding media that are present in thin sections (Muller and Engel, 2001). Thus, these features limit the ability of electron microscopic measurements to determine mass per unit area on an absolute scale, although quantitative microscopy can make direct comparisons as long as the assumptions are reasonably met that the stain density is related to protein density and the stains are not selective. At present there are no quantitative studies that directly relate en bloc stain intensity to protein density, although there is substantial evidence that there is a direct relationship in standard thin section analysis (Glauert and Lewis, 1998; Hayat 2000). It is well documented that crystallin proteins undergo age-related modifications, which can potentially alter their ability to uniformly take up stain. The nearly uniform staining of cytoplasm and most MLBs supports the conclusion that selective staining is minimal. Moreover, it is expected that all of these factors similarly influence the MLB cores and the adjacent cytoplasm such that quantitative comparisons can be made to derive accurate RI ratios.

\section{Conclusions}

A novel and relatively easy method was developed for determining RI values of MLB interiors compared to adjacent cytoplasm directly from transmission electron micrographs of thin sections. MLB RIs have been measured in the range 1.35-1.53 (mean $\pm \mathrm{SD}=$ $1.461 \pm 0.035$, median $=1.450, \mathrm{n}=61$ particles), assuming the cytoplasmic RI to be 1.42 . The low refractive index shells that develop around mature MLBs have a minor effect on the total scattering. The most important factor is the MLB/cytoplasm RI ratio. Mie theory predicts that forward scattering, from particles in the range $2-4 \mu \mathrm{m}$ in diameter with internal refractive indices between $1.49-1.53$, can be more than $30 \%$. This excessive forward scattering influences macular image formation and would be valuable additional information for ophthalmological evaluations of cataract patients.

\section{Acknowledgments}

We thank Mr. Kenji Leonard, Mr. Harold Mekeel, Ms. Lauren Averette, Ms. Mariko Weber for their technical assistance. We are grateful to Dr. W. Craig Fowler, the NC Eye and Tissue Bank, and surgeons at the LV Prasad Eye Institute for providing lens specimens. This work was supported in part by the NIH grant EY008148 (MJC) and core grant EY005722 (Duke).

\section{References}

Al-Ghoul KJ, Costello MJ. Fiber cell morphology and cytoplasmic texture in cataractous and normal human lens nuclei. Curr. Eye Res 1996;15:533-542. [PubMed: 8670754]

Bahr GF, Engler WF, Mazzone HM. Determination of the mass of viruses by quantitative electron microscopy. Q. Rev. Biophys 1976;9:459-489. [PubMed: 189345]

Bahr GF, Zeitler E. The Determination of the Dry Mass in Populations of Isolated Particles. Lab Invest 1965;14:955-977. [PubMed: 14317060]

Benedek GB. Cataract as a protein condensation disease: the Proctor Lecture. Invest. Ophthalmol. Vis. Sci 1997;38:1911-1921. [PubMed: 9331254] 
Birnie GD, MacPhail E, Rickwood D. Isopycnic sedimentation of DNA in metrizamide: the effect of low concentrations of ions on buoyant density and hydration. Nucleic Acids Res 1974;1:919-925. [PubMed: 10793724]

Charney E, Brackett FS. The spectral dependence of scattering from a spherical alga and its implications for the state of organization of the light-accepting pigments. Arch. Biochem. Biophys 1961;92:1-12. [PubMed: 13692507]

Costello MJ, Johnsen S, Gilliland KO, Freel CD, Fowler WC. Predicted Light Scattering from Particles Observed in Human Age-Related Nuclear Cataracts Using Mie Scattering Theory. Invest. Ophthalmol. Vis. Sci 2007;48:303-312. [PubMed: 17197547]

Costello MJ, Johnsen S, Metlapally S, Gilliland KO, Ramamurthy B, Krishna PV, Balasubramanian D. Ultrastructural analysis of damage to nuclear fiber cell membranes in advanced age-related cataracts from India. Exp. Eye Res 2008;87:147-158. [PubMed: 18617164]

Costello, MJ.; Kuszak, AJ. The Types, Morphology and Causes of Cataracts. In: Garner, A.; Klintoworth, GK., editors. Pathobiology of Ocular Disease. Informa Healthcare; New York, NY, USA: 2008. p. 469-494.

Datiles MB 3rd, Ansari RR, Reed GF. A clinical study of the human lens with a dynamic light scattering device. Exp. Eye. Res 2002;74:93-102. [PubMed: 11878822]

Freel CD, Gilliland KO, Lane C. Wesley, Giblin FJ, Costello MJ. Fourier analysis of cytoplasmic texture in nuclear fiber cells from transparent and cataractous human and animal lenses. Exp. Eye. Res 2002;74:689-702. [PubMed: 12126943]

Glauert, AM.; Lewis, PR. Biological specimen preparation for transmission electron microscopy. Princeton University Press; Princeton, NJ, USA: 1998.

Gilliland KO, Freel CD, Johnsen S, Fowler W. Craig, Costello MJ. Distribution, spherical structure and predicted Mie scattering of multilamellar bodies in human age-related nuclear cataracts. Exp. Eye. Res 2004;79:563-576. [PubMed: 15381040]

Gilliland KO, Freel CD, Lane CW, Fowler WC, Costello MJ. Multilamellar bodies as potential scattering particles in human age-related nuclear cataracts. Mol. Vis 2001;7:120-130. [PubMed: 11435998]

Gilliland KO, Johnsen S, Metlapally S, Costello MJ, Ramamurthy B, Krishna PV, Balasubramanian D. Mie light scattering calculations for an Indian age-related nuclear cataract with a high density of multilamellar bodies. Mol. Vis 2008;14:572-582. [PubMed: 18385793]

Hayat, MA. The Principles and Techniques for Electron Microscopy. 4th ed. Cambridge University Press; Cambridge, UK: 2000.

Herrmann H, Haner M, Brettel M, Muller SA, Goldie KN, Fedtke B, Lustig A, Franke WW, Aebi U. Structure and assembly properties of the intermediate filament protein vimentin: the role of its head, rod and tail domains. J. Mol. Biol 1996;264:933-953. [PubMed: 9000622]

Jones CE, Atchison DA, Meder R, Pope JM. Refractive index distribution and optical properties of the isolated human lens measured using magnetic resonance imaging (MRI). Vision Res 2005;45:2352-2366. [PubMed: 15979462]

Kammerer RA, Kostrewa D, Zurdo J, Detken A, Garcia-Echeverria C, Green JD, Muller SA, Meier BH, Winkler FK, Dobson CM, Steinmetz MO. Exploring amyloid formation by a de novo design. Proc. Natl. Acad. Sci. U S A 2004;101:4435-4440. [PubMed: 15070736]

Krzyzanek V, Muller SA, Engel A, Reichelt R. MASDET-A fast and user-friendly multiplatform software for mass determination by dark-field electron microscopy. J. Struct. Biol 2009;165:7887. [PubMed: 19041401]

Lamvik MK. Muscle thick filament mass measured by electron scattering. J. Mol. Biol 1978;122:5568. [PubMed: 671551]

Lamvik MK, Davilla S. Calibration methods for quantitative image processing in electron microscopy. J. Electron. Microsc. Tech 1989;11:97-101. [PubMed: 2709141]

Matsuzaki K, Murase O, Sugishita K, Yoneyama S, Akada K, Ueha M, Nakamura A, Kobayashi S. Optical characterization of liposomes by right angle light scattering and turbidity measurement. Biochim. Biophys. Acta 2000;1467:219-226. [PubMed: 10930524]

McGinty SJ, Truscott RJ. Presbyopia: the first stage of nuclear cataract? Ophthalmic Res 2006;38:137-148. [PubMed: 16397406] 
Metlapally S, Costello MJ, Gilliland KO, Ramamurthy B, Krishna PV, Balasubramanian D, Johnsen S. Analysis of nuclear fiber cell cytoplasmic texture in advanced cataractous lenses from Indian subjects using Debye-Bueche theory. Exp. Eye Res 2008;86:434-444. [PubMed: 18191834]

Mosesson MW, Hainfeld J, Wall J, Haschemeyer RH. Identification and mass analysis of human fibrinogen molecules and their domains by scanning transmission electron microscopy. J. Mol. Biol 1981;153:695-718. [PubMed: 7338923]

Muller SA, Engel A. Structure and mass analysis by scanning transmission electron microscopy. Micron 2001;32:21-31. [PubMed: 10900377]

Muller SA, Goldie KN, Bürki R, Häring R, Engel A. Factors influencing the precision of quantitative scanning electron microscopy. Ultramicroscopy 1992;46:317-334.

Pierscionek BK. Refractive index contours in the human lens. Exp. Eye. Res 1997;64:887-893. [PubMed: 9301469]

Pierscionek BK, Smith G, Augusteyn RC. The refractive increments of bovine alpha-, beta-, and gamma-crystallins. Vision Res 1987;27:1539-1541. [PubMed: 3445487]

Tardieu A, Veretout F, Krop B, Slingsby C. Protein interactions in the calf eye lens: interactions between beta-crystallins are repulsive whereas in gamma-crystallins they are attractive. Eur. Biophys. J 1992;21:1-12. [PubMed: 1516556]

Taylor VL, Costello MJ. Fourier analysis of textural variations in human normal and cataractous lens nuclear fiber cell cytoplasm. Exp. Eye. Res 1999;69:163-174. [PubMed: 10433853]

Truscott RJ. Age-related nuclear cataract-oxidation is the key. Exp. Eye. Res 2005;80:709-725. [PubMed: 15862178]

van den Berg TJ. Light scattering by donor lenses as a function of depth and wavelength. Invest. Ophthalmol. Vis. Sci 1997;38:1321-1332. [PubMed: 9191595]

Walz T, Smith BL, Zeidel ML, Engel A, Agre P. Biologically active two-dimensional crystals of aquaporin CHIP. J. Biol. Chem 1994;269:1583-1586. [PubMed: 8294400]

Zeitler E, Bahr GF. A Photometric Procedure for Weight Determination of Submicroscopic Particles Quantitative Electron Microscopy. J. Appl. Phys 1962;33:847-853.

Exp Eye Res. Author manuscript; available in PMC 2011 December 1. 


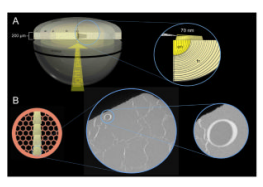

Figure 1. 

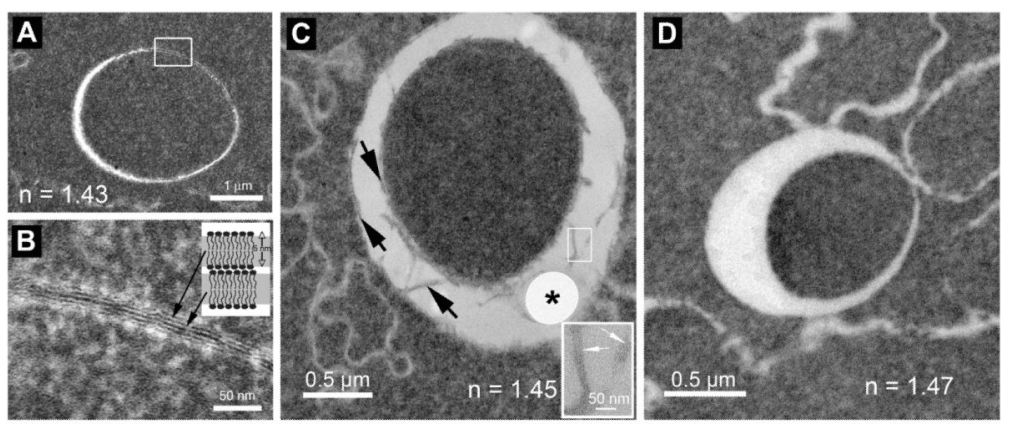

Figure 2. 


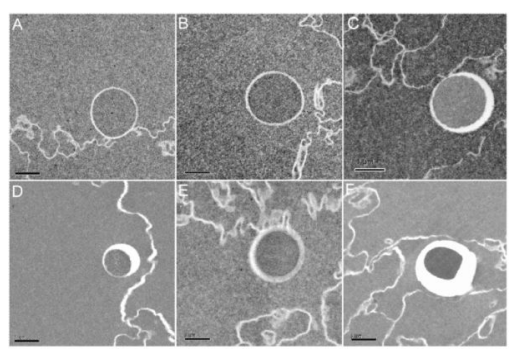

Figure 3. 


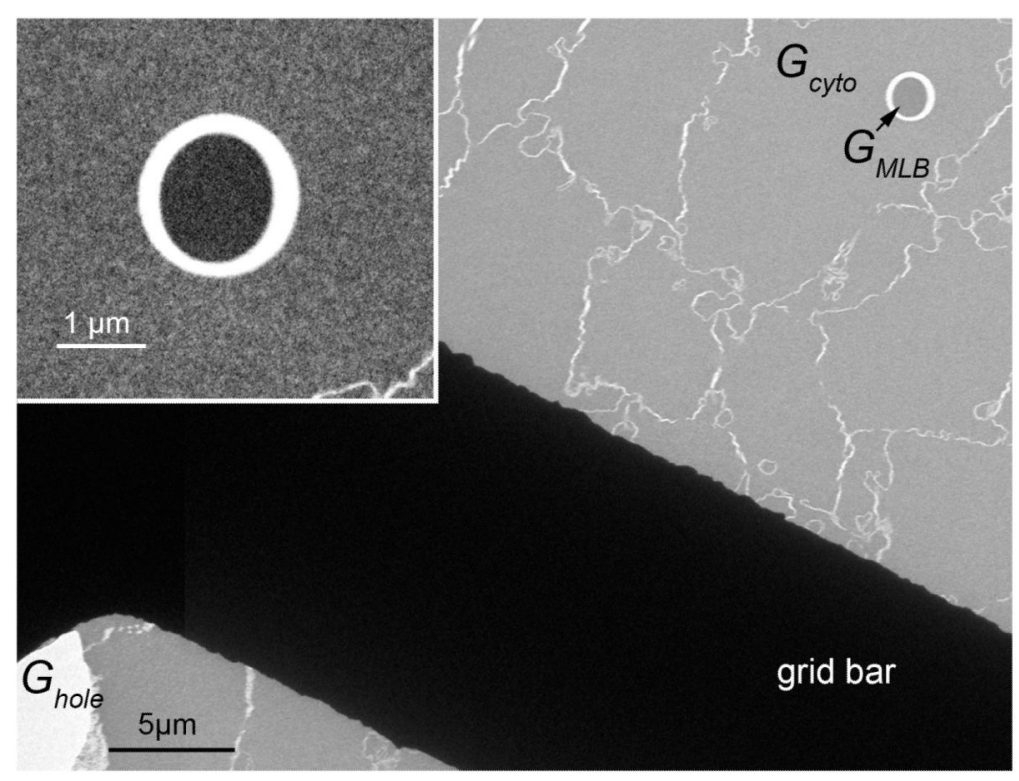

Figure 4. 


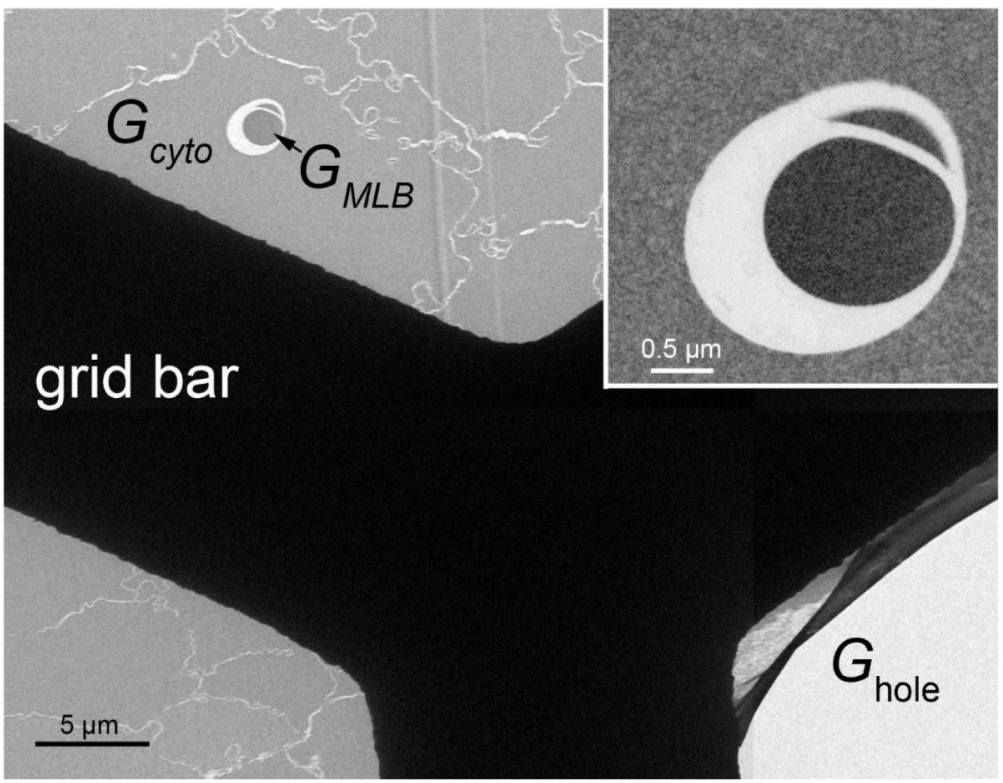

Figure 5. 


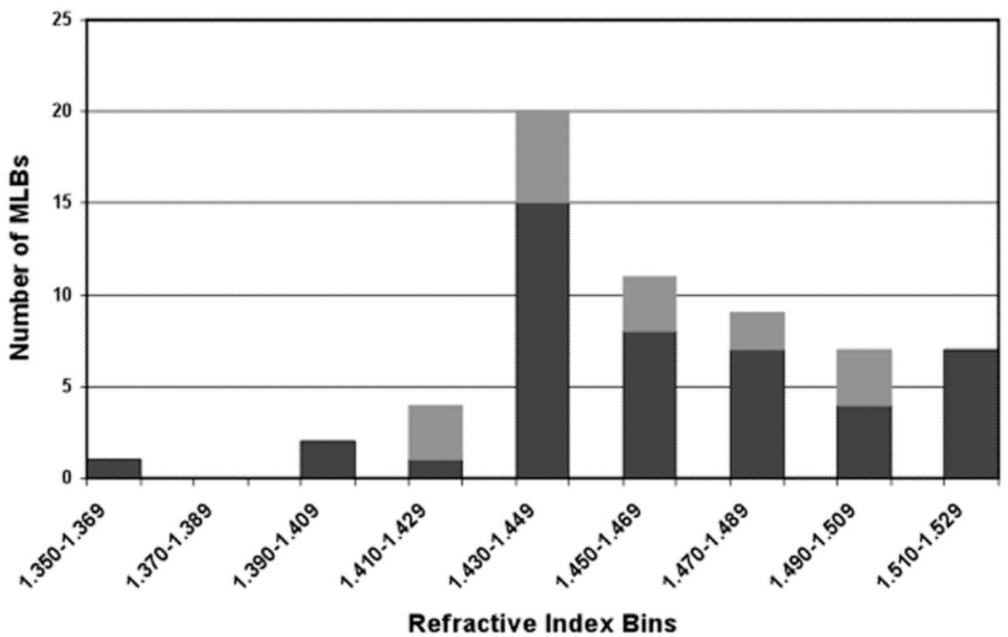

Figure 6. 
Costello et al.

Page 19
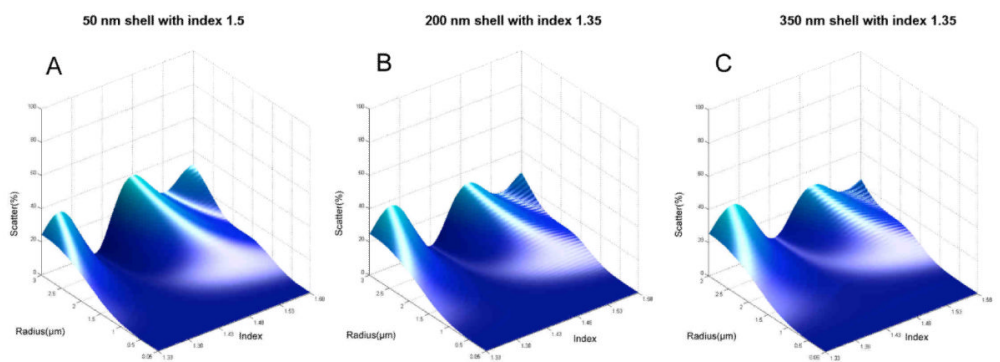

Figure 7.

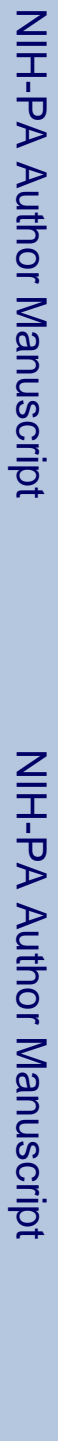

Exp Eye Res. Author manuscript; available in PMC 2011 December 1. 


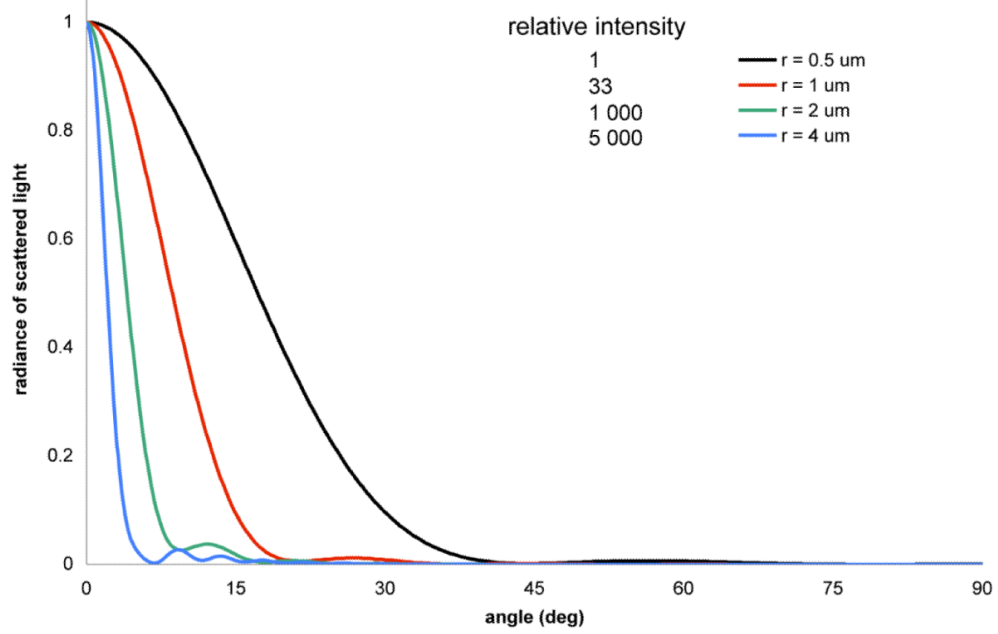

Figure 8. 